\title{
Educación Superior: Pandemia COVID-19
}

\author{
Paloma Valdivia Vizarreta \\ https://orcid.org/0000-0003-1499-5478 \\ Universitat Autònoma de Barcelona, España \\ Departamento de Teorías de la Educación y Pedagogía Social
}

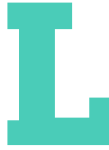

a situación de emergencia sanitaria llevó a las universidades que imparten formación presencial a adaptarse a la virtualidad. Durante este periodo sus acciones han sido cruciales para salvaguardar el progreso educativo (Wang et al., 2020), dentro de sus posibilidades, se han esforzado en hacerlo de manera ágil y rápida, no planificada. Han utilizado los recursos tecnológicos con los que contaban, han coordinado una intensa labor de reprogramación, aprendizaje y adaptación por parte del profesorado y alumnado (Crawford, 2020). Todo ello, a la par de la conciliación, entre estos nuevos escenarios de aprendizaje en casa y su vida familiar.

Antes del confinamiento, ya existían demandas de cambio en la universidad, como por ejemplo la necesidad del desarrollo de competencias digitales de los docentes (Instefjord and Munthe, 2015; Redecker, 2017; Reisoğlu and Çebi, 2020); durante este periodo se ha evidenciado un acelerado y forzado proceso de adopción y adaptación de las tecnologías. El profesorado han tenido que asumir las posibilidades digitales existentes hace ya algún tiempo, entre ellas podemos destacas como beneficios el aprendizaje distribuido y ubicuo, es decir, la flexibilidad del tiempo y lugar, facilidad para organizar y gestionar las tareas de estudio hasta la capacidad de reproducir y revisar los materiales didácticos y aprender en formas más visuales (Henderson et al., 2015).

La brecha digital previa al confinamiento, ha demostrado que no solo era por parte del alumnado y profesorado, si no de las universidades. Para Bao (2020), durante el Covid-19, los desafíos de las universidades se centraron en contar con una infraestructura de red tecnológica-educativa confiable para migrar y adaptar los cursos, alinear las competencias del plan de estudios, repensar la evaluación, ampliar la capacitación del profesorado y tener un plan de contingencia frente a incidentes inesperados. 
El bienestar y las competencias socioemocionales ha sido otro reto de las universidades (Hadar et al., 2020), relacionado con ofrecer servicios de apoyo instantáneo a sus profesores/as y estudiantes, informar sobre sus propuestas de aprendizajes y la aplicación de las políticas sanitarias. Crawford (2020) añade incluir el apoyo a estudiantes internacionales separados de amigos y familiares.

Durante este periodo, también han cobrado relevancia temas relacionados con la ética (Noguera et al., 2020), como la privacidad en las videoconferencias, los derechos de imagen y de propiedad intelectual, las evaluaciones con sistemas biométricos y programas informáticos anti-plagio.

¿Cómo se superará esta crisis mundial en educación? ¿Qué puede aportar la universidad? ¿Se mantendrá el entusiasmo tecnopedagógico cuando se detenga la crisis? ¿Qué está cambiando en este diseño de aprendizaje? ¿Estamos preparados para asegurar la docencia universitaria en una nueva situación de confinamiento? ¿Será sostenible la formación permanente del profesorado? ¿Cómo lograr el equilibrio entre calidad de enseñanza, recursos y apropiación tecnológica? ¿Después del Covid se propone un nuevo paradigma?

Es necesario dedicar tiempo a la reflexión desde la acción, y elabora propuestas globales y locales a estos cuestionamientos (Rapanta et al., 2020). Por ello, consideramos que los resultados de las investigaciones de este monográfico aportan una mirada internacional en línea de varios de los desafíos mencionados. A continuación se detalla cada uno de ellos, agrupados por las temáticas que desarrollan.

Al realizar el análisis de la educación superior en la cuarentena, Franklin Américo Canaza-Choque desde Perú, pone énfasis en la desigualdad educativa, la pobreza, la exclusión social, racial y de género.

Celio Luis Acosta Álvarez, Diosvany Ortega González y Yosefint Díaz Cruz nos detallan el modelo implementado en la Universidad de San Pedro Sula en Honduras, de educación presencial con mediación virtual que se organizó por fases claramente definidas así como la evaluación de la satisfacción del profesorado y del alumnado.

Se destaca la necesidad de un cambio educativo hacia modelos y prácticas más flexibles que puedan responder a la complejidad y la imprevisibilidad de los sistemas rápidos e interconectados de hoy y en una sociedad todavía frágil. En ese sentido, Lorena Elizabeth Araujo Silva, Juan Felipe Ochoa Mogrovejo y Catalina Vélez Verdugo analizan los desafíos de la universidad ecuatoriana previos a la pandemia y su transición a la situación actual de enseñanza remota de emergencia.

Las tecnologías en línea pueden servir como catalizadores para que los profesores experimenten cosas nuevas, explorar alternativas creativas y reflexionar sobre sus propias prácticas (Rapanta et al., 2020). Así lo demuestran Marisa S. Castro, Mariela L Paz y Eliana M. Cela, en su artículo. Comparten las experiencias docentes en una universidad Argen- 
tina, destacando el estrecho contacto con el alumnado, las estrategias metodológicas, su compromiso y predisposición.

Además de la adaptación metodológica durante este periodo de emergencia, la evaluación fue un gran reto. No solo por el cambio de escenarios, acompañamiento si no por la fiabilidad de los aprendizajes. Sobre este tema Laura Cañadas sintetiza las propuestas y oportunidades de evaluación formativa. Pilar Rodríguez Morales y Mario Luzardo Verde analizan cómo asegurar evaluaciones válidas y detectar falseamiento de las pruebas a distancia síncronas.

La internacionalización y la movilidad estudiantil ha crecido y crecerá en los años siguientes según el informe Doctoral education in Europe today: approaches and institutional structures (Hasgall et al., 2019). Pamela Gómez López desarrolla la siguiente pregunta ¿qué sucederá con la internacionalización de la educación superior y la movilidad estudiantil después del COVID-19? Durante este periodo de incertidumbre, el alumnado desestimó matricularse en formaciones pensadas para ser presenciales virtuales.

Varias universidades aprovecharon esta situación para incrementar su oferta de formación permanente, utilizando recursos como los MOOC, seminarios virtuales en sus canales de vídeos. Diversificaron de estas y otras maneras sus posibilidades de aprendizaje no formal o informal. Estas nuevas y amplias posibilidades plantean nuevos retos al reconocimiento de estos estudios. Dentro de las nuevas opciones de certificación o acreditación de los aprendizajes Ismael Canales-Negrón analiza 20 investigaciones de insignias digitales en programas de educación continua profesional, una propuesta interesante, que desde hace algunos años se viene utilizando como alternativa, pero aún no se ha masificado su uso.

Otro tema, que se ha incrementado durante este periodo es la preocupación por la proliferación de información engañosa sobre el Covid-19, rumores y mitos. Ello ha llevado a los gobiernos de todo el mundo a instituir varias medidas para detener su flujo (Rodrigues and $\mathrm{Xu}, 2020$ ). Wendy Villafranqui Cabanillas evidencia la validez que se le da a las noticias falsas y propone un método de trabajo para las entidades de formación docente focalizado en la interacción con textos de investigación científica.

Dentro de la preocupación de la universidad por el bienestar y las competencias socioemocionales, Ricardo Jorquera Gutiérrez y Fernando Herrera Gallardo analizan la salud mental de funcionariado de una universidad chilena desde el estrés, la depresión y la ansiedad unida a los desafíos a los que se han presentado en el contexto de la COVID-19. Desde un análisis predictivo, Rony Prada-Chapoñan, Jhonatan S. Navarro-Loli y Sergio Dominguez-Lara evidencian los factores del predicen el agotamiento emocional de forma significativa en estudiantes peruanos.

Finalmente, las decisiones de las universidades continuaran cambiando significativamente en los próximos meses, los gobiernos dictando diversas directivas y la sociedad 
asumiendo nuevos modelos de vida. En este contexto, las universidades deben liderar esta transición, por un lado, para apoyar a una sociedad que necesita permanecer en casa de manera intermitente y por otro, mantener su nivel de calidad, productividad e investigación en el corto y potencialmente el mediano plazo.

\section{Referencias}

Bao, W. (2020). COVID 19 and online teaching in higher education: A case study of Peking University. Human Behavior and Emerging Technologies, 2(2), 113-115. https://doi.org/10.1002/hbe2.191

Crawford, J. (2020). COVID-19: 20 countries' higher education intra-period digital pedagogy responses. Journal of Applied Learning \& Teaching, 3(1), 09-28. https://doi.org/10.37074/jalt.2020.3.1.7

Hadar, L. L., Ergas, O., Alpert, B., and Ariav, T. (2020). Rethinking teacher education in a VUCA world: student teachers' social-emotional competencies during the Covid-19 crisis. European Journal of Teacher Education, 1-14. https://doi.or g/10.1080/02619768.2020.1807513

Hasgall, A., Saenen, B., and Borrell-Damian, L. (2019). Doctoral Education in Europe Today: Approaches and Institutional Structures. Survey. In European University Association. European University Association.

Henderson, M., Selwyn, N., and Aston, R. (2015). What works and why? Student perceptions of 'useful' digital technology in university teaching and learning. Studies in Higher Education, 42(8), 1567-1579. https://doi.org/10.1080/03075079 .2015 .1007946

Instefjord, E., and Munthe, E. (2015). Preparing pre-service teachers to integrate technology: an analysis of the emphasis on digital competence in teacher education curricula. European Journal of Teacher Education, 39(1), 77-93. https://doi. org/10.1080/02619768.2015.1100602

Noguera, I., Sala Roca, J., and Valdivia, P. (2020). Docència a distància d'emergència. https://ddd.uab.cat/record/231272

Rapanta, C., Botturi, L., Goodyear, P., Guàrdia, L., and Koole, M. (2020). Online University Teaching During and After the Covid-19 Crisis: Refocusing Teacher Presence and Learning Activity. Postdigital Science and Education, 1-23. https:// doi.org/10.1007/s42438-020-00155-y

Redecker, C. (2017). European framework for the digital competence of educators: DigCompEdu. In Joint Research Centre (JRC) Science for Policy report. https://doi.org/10.2760/159770

Reisoğlu, İ., and Çebi, A. (2020). How can the digital competences of pre-service teachers be developed? Examining a case study through the lens of DigComp and DigCompEdu. Computers and Education, 156, 103940. https://doi.org/10.1016/j.compedu.2020.103940

Rodrigues, U. M., and Xu, J. (2020). Regulation of COVID-19 fake news infodemic in China and India. Media International Australia, 177(1), 125-131. https://doi.org/10.1177/1329878X20948202

Wang, C., Cheng, Z., Yue, X.-G., and McAleer, M. (2020). Risk Management of COVID-19 by Universities in China. Journal of Risk and Financial Management, 13(2), 36. https://doi.org/10.3390/jrfm13020036

RIDU / Revista Digital de Investigación en Docencia Universitaria / e-ISSN: 2223-2516

(c) Los autores. Este artículo es publicado por la Revista Digital de Investigación en Docencia Universitaria del Área de Institutional Research and Effectiveness de la Dirección de Aseguramiento de la Calidad, Universidad Peruana de Ciencias Aplicadas. Este es un artículo de acceso abierto, distribuido bajo los términos de la LicenciaCreativeCommons Atribución-CompartirIgual 4.0 Internacional.( http://creativecommons.org/licenses/by-sa/4.0/), que permite el uso no comercial, distribución y reproducción en cualquier medio, siempre que la obra original sea debidamente citada. 